\title{
Measurement of antineutrino oscillation with the full detector configuration at Daya Bay
}

\author{
Marco GRASSI ${ }^{* \dagger}$ \\ IHEP, Chinese Academy of Sciences, 19B Yuquanlu, Beijing, P.R. China \\ E-mail: mgrassi@ihep.ac.cn \\ on behalf of the DAYA BAY Collaboration
}

In this poster, we present the latest measurement of electron antineutrino disappearance using the fully constructed Daya Bay Reactor Neutrino Experiment. A total exposure of $6.9 \times 10^{5} \mathrm{GW}_{\text {th }}$ ton days was achieved in November 2013 after 617 day of data taking. The most precise estimates to date of the neutrino mass and mixing parameters $\left|\Delta \mathrm{m}_{e e}^{2}\right|$ and $\sin ^{2} 2 \theta_{13}$ was obtained with an analysis of the relative antineutrino rates and energy spectra between detectors. The value of the two parameters was found to be $\sin ^{2} 2 \theta_{13}=0.084 \pm 0.005$ and $\left|\Delta \mathrm{m}_{e e}^{2}\right|=(2.42 \pm 0.11) \times 10^{-3} \mathrm{eV}^{2}$. This poster focuses in particular on describing how improvements in the calibration and in the energy response model contributed to achieve this result.

XXVII International Symposium on Lepton Photon Interactions at High Energies

17-22 August 2015

Ljubljana, Slovenia

\footnotetext{
* Speaker.

${ }^{\dagger}$ MG is supported by the Chinese Academy of Sciences President's International Fellowship Initiative, grant 2015 PM007.
} 


\section{Experimental Apparatus and Methods}

Neutrino flavour oscillation due to the mixing angle $\theta_{13}$ has been observed using reactor antineutrinos $\left(\bar{v}_{e}\right)[1,2,3]$ and accelerator neutrinos [4, 5]. The DAYA BAY experiment previously reported the discovery of a non-zero value of $\sin ^{2} 2 \theta_{13}$ by observing the disappearance of reactor antineutrinos over kilometre distances [1], and the first measurement of the effective mass splitting $\left|\Delta m_{e e}^{2}\right|$ via the distortion of the $\bar{v}_{e}$ energy spectrum [6]. This poster presents new results with larger statistics and significant improvements in energy calibration and background reduction published in [7].

The DAYA BAY experiment consists of eight functionally identical antineutrino detectors (ADs) hosted in three underground experimental halls (EHs), detecting reactor $\bar{v}_{e}$ via inverse beta decay (IBD) reactions. EH1 and EH2 are respectively located at short distance from the Daya Bay and Ling Ao reactor cores, and they both host two ADs. EH3 is at $1.6 \mathrm{~km}$ distance from the cores and hosts 4 ADs. Each EH is equipped with a muon detector system to tag and veto cosmic muons, consisting of a layer of resistive plate chambers (RPCs) and a water Cherenkov detector, in which the ADs are immersed.

Each AD has three nested cylindrical volumes separated by concentric acrylic vessels, while the outermost vessel is made of stainless steel. The innermost volume holds 20 ton of gadoliniumdoped liquid scintillator (Gd-LS) that serves as the antineutrino target. The middle volume is called the gamma catcher and is filled with 21 ton of undoped liquid scintillator (LS) for detecting gamma rays that escape the target volume. The outer volume contains 37 ton of mineral oil (MO) to provide optical homogeneity and to shield the inner volumes from background radiation. The outer volume also hosts 192 eight-inch PMTs facing the target volume.

IBDs are selected by exploiting the prompt-delayed time structure of the event, where the positron annihilates soon after its production (prompt energy), while the neutron gets captured on gadolinium with a mean capture time of $\sim 30 \mu$ s (delayed energy). In order for an event to be selected, the difference between the two energy depositions is required to be between 1 and $200 \mu$ s.

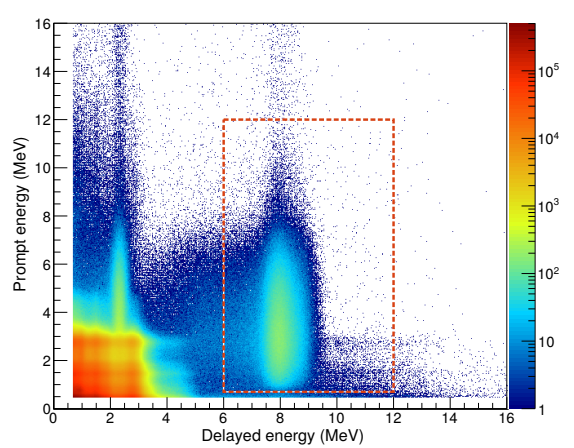

Figure 1. IBD prompt/delayed energy spectrum. The dashed line shows the energy-based selection criteria.

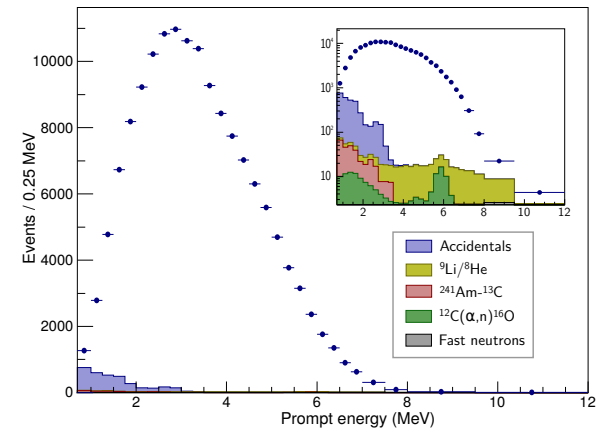

Figure 2. Prompt energy spectrum of IBD candidates in EH3 together with the subtracted background components. The inset shows the same plot in logarithmic scale. 


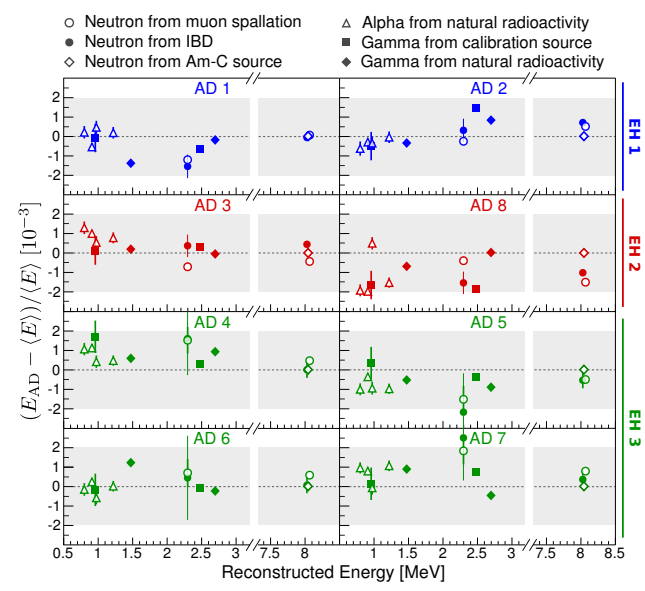

Figure 3. Comparison of the reconstructed energy between antineutrino detectors for a variety of calibration references. $\mathrm{E}_{\mathrm{AD}}$ is the reconstructed energy determined using each AD, and $\langle E\rangle$ is the 8-detector average. Error bars are statistical only, and systematic variations between detectors for all calibration references are $<0.2 \%$. The $\sim 8 \mathrm{MeV}$ n-Gd capture gamma peaks from Am-C sources are used to define the energy scale of each detector, and hence show zero deviation.

Additional energy selection criteria require the prompt energy to be in the $0.7-12 \mathrm{MeV}$ range, and the delayed energy to be in the 6-12 MeV range, as shown in Fig. 1. In order to suppress cosmogenic products, IBD candidates are rejected if the delayed signal occurs soon after a muon trigger, where the vetoed time window can range from $600 \mu \mathrm{s}$ to $1 \mathrm{~s}$ according the amount of energy deposited in the detector. The prompt energy spectrum of selected events, together with the five major sources of background (fast neutrons of cosmogenic origin, correlated $\beta$-n decays from cosmogenic ${ }^{9} \mathrm{Li}$ and ${ }^{8} \mathrm{He},{ }^{13} \mathrm{C}(\alpha, \mathrm{n}){ }^{16} \mathrm{O}$ reactions, single neutrons from the Am- $\mathrm{C}$ calibration sources and accidental coincidences), are shown in Fig. 2.

We use the prompt energy as a proxy for the incident $\bar{v}_{e}$ energy, and any difference in the energy response between ADs affects the estimation of $\left|\Delta \mathrm{m}_{e e}^{2}\right|$. The detector energy scale is calibrated using Am-C neutron sources deployed at the detector centre, with the $\sim 8 \mathrm{MeV}$ peaks from neutrons captured on Gd aligned across all eight ADs. The time variation and the position dependence of the energy scale are corrected using the $2.5 \mathrm{MeV}$ gamma-ray peak from ${ }^{60} \mathrm{Co}$ calibration sources. The uncertainty associated to any residual difference in the energy response is evaluated by comparing several calibration reference points across all ADs, as shown in Fig. 3. Such reference points are: (I) ${ }^{68} \mathrm{Ge},{ }^{60} \mathrm{Co}$ and Am-C calibration sources placed at detector centre, (II) Gd-captured neutrons from IBD and muon spallation, distributed nearly uniformly throughout the Gd-LS region, (III) neutrons being captured on ${ }^{1} \mathrm{H}$, intrinsic $\alpha$ particles from polonium and radon decays, and gammas from ${ }^{40} \mathrm{~K}$ and ${ }^{208} \mathrm{Tl}$ decays, all being distributed inside and outside of the target volume. Events from all the calibration samples are required to have the reconstructed vertex within the Gd-LS region. However, since the spatial distribution of each calibration sample varies, the energy difference shown in Fig. 3 incorporates also deviations in spatial response between detectors. The resulting uncorrelated relative uncertainty of the energy scale is $0.2 \%$.

The detector energy response is known to be non-linear. The main causes of this behaviour are: (I) a particle-dependent non-linear light yield of the scintillator, and (II) a charge-dependent non-linearity in the PMT readout electronics, each being at the level of $10 \%$ within the IBD energy range. We model the non-linear response with a semi-empirical model, which can be factorised into two independent components: $f_{\text {scintillator }}\left(k_{B}, k_{C}\right)$-where the two free parameters are the Birks' constant $k_{B}$ and the fraction of Cherenkov light contributing to the total light yield $k_{C}$-, and $f_{\text {electronics }}(\alpha, \tau)$-where $\alpha$ and $\tau$ are respectively the amplitude and the scale of the exponential 
describing the electronics non-linearity.

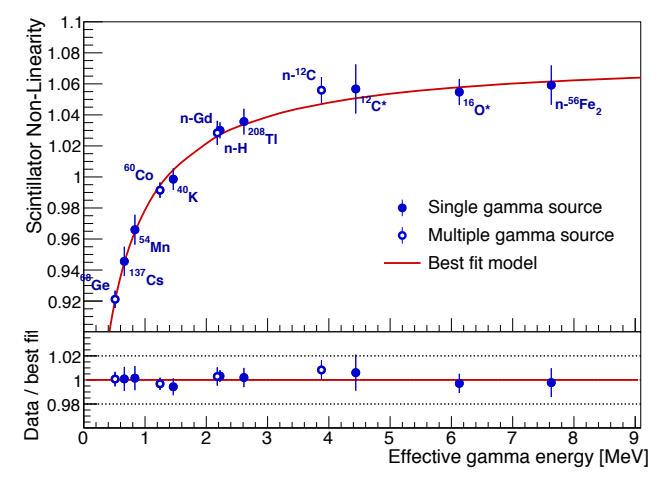

Figure 4. The scintillator non-linearity resulting from the best-fit parameters of the overall energy response model, compared to deployed and intrinsic gamma calibration sources.

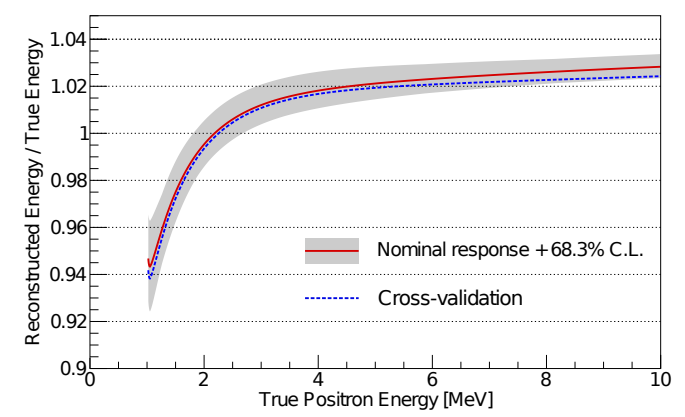

Figure 5. Solid curve: Estimated energy response of the detectors to positrons, based on gamma rays from both deployed and intrinsic sources, as well as electrons from ${ }^{12} \mathrm{~B} \beta$ decays. Dashed curve: Validation model based on ${ }^{12} \mathrm{Bi}$, ${ }^{214} \mathrm{Bi},{ }^{208} \mathrm{Tl} \beta+\gamma$ spectra together with the 53$\mathrm{MeV}$ edge in the Michel electron spectrum.

The values of these four free parameters are obtained from an unconstrained $\chi^{2}$ fit to several calibration datasets, namely 12 gamma lines by both artificial sources deployed at detector centre and naturally occurring sources, and the continuous $\beta$ decay spectrum of ${ }^{12} \mathrm{~B}$ resulting from muon spallation events in the Gd-LS volume. The comparison of the best fit model against gamma data is shown in Fig. 4, while the nominal positron response derived from the best fit parameters is represented by the solid curve in Fig. 5. The depicted uncertainty band represents other response functions consistent with the fitted calibration data within a $68.3 \%$ C.L. The positron response is further validated with the $53-\mathrm{MeV}$ cutoff in the decay electron spectrum from muon decay at rest, and with the continuous $\beta+\gamma$ spectra from natural bismuth and thallium decays. This additional model —represented by the blue dashed curve in Fig. 5- falls within the 1- $\sigma$ contour of the nominal positron response curve, improving our confidence in the characterization of the absolute energy response of the detectors.

\section{Results}

Neutrino oscillation parameters are measured using the L/E-dependent disappearance of $\bar{v}_{e}$, as given by the survival probability

$$
P \simeq 1-\cos ^{4} \theta_{13} \sin ^{2} 2 \theta_{12} \sin ^{2} \frac{1.267 \Delta m_{21}^{2} L}{E}-\sin ^{2} 2 \theta_{13} \sin ^{2} \frac{1.267 \Delta m_{e e}^{2} L}{E} .
$$

Here $\mathrm{E}$ is the energy in $\mathrm{MeV}$ of the $\bar{v}_{e}, \mathrm{~L}$ is the distance in meters from its production point, $\theta_{12}$ is the solar mixing angle, and $\Delta m_{12}^{2}=m_{2}^{2}-m_{1}^{2}$ is the mass-squared difference of the first two neutrino mass eigenstates in $\mathrm{eV}^{2}$. Since recent measurements of the IBD positron energy spectrum disagree with models of reactor $\bar{v}_{e}$ emission $[3,8,9]$, to measure the neutrino oscillation parameters we employ a technique predicting the signal in the far hall based on measurements 
obtained in the near halls. This allows us to minimise the result's dependence on models of the reactor antineutrino flux (more information can be found in [7]). Out of this approach, we obtain $\sin ^{2} 2 \theta_{13}=0.084 \pm 0.005$ and $\Delta \mathrm{m}_{e e}^{2}=(2.42 \pm 0.11) \times 10^{-3} \mathrm{eV}^{2}$, with $\chi^{2} / \mathrm{NDF}=134.6 / 146$. Under the normal (inverted) hierarchy assumption, $\left|\Delta \mathrm{m}_{e e}^{2}\right|$ yields $\Delta m_{32}=(2.37 \pm 0.11) \times 10^{-3} \mathrm{eV}^{2}$ $\left(\Delta m_{32}=-(2.47 \pm 0.11) \times 10^{-3} \mathrm{eV}^{2}\right)$. This result is consistent with and of compatible precision to measurements obtained from accelerator $v_{\mu}$ and $\bar{v}_{\mu}$ disappearance [11, 12]. The reconstructed positron energy spectrum observed in the far site is compared in Fig. 6 with the expectation based on the near-site measurements. The total uncertainties of both $\sin ^{2} 2 \theta_{13}$ and $\left|\Delta \mathrm{m}_{e e}^{2}\right|$ are dominated by statistics. The most significant systematic uncertainties for $\sin ^{2} 2 \theta_{13}$ are due to the relative detector efficiency, reactor power, relative energy scale, and ${ }^{9} \mathrm{Li} /{ }^{8} \mathrm{He}$ background. The systematic uncertainty in $\left|\Delta \mathrm{m}_{e e}^{2}\right|$ is dominated by uncertainty in the relative energy scale.

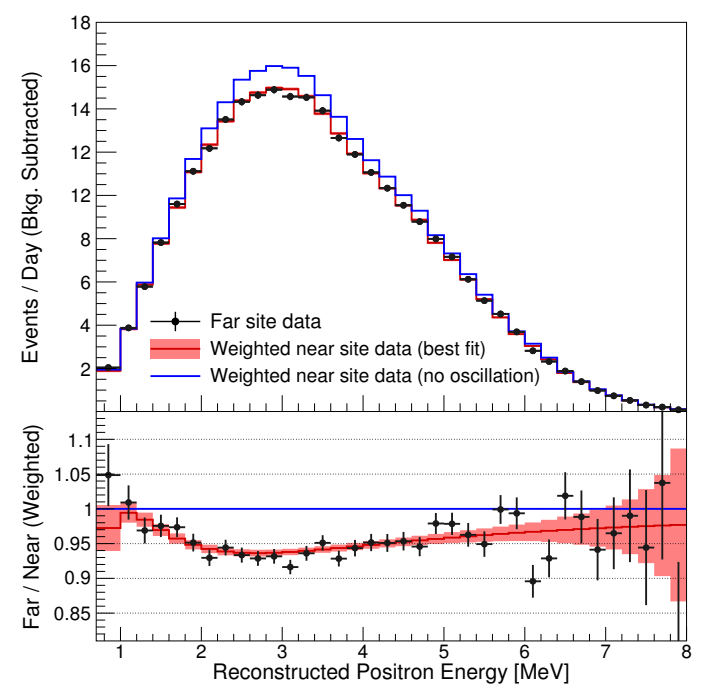

Figure 6. Top: Background-subtracted reconstructed positron energy spectrum observed in the far site (black points), as well as the expectation derived from the near sites excluding (blue line) or including (red line) our best estimate of oscillation. The spectra were efficiency corrected and normalised to one day of live time. Bottom: Ratio of the spectra to the no-oscillation case. The error bars show the statistical uncertainty of the far site data. The shaded area includes the systematic and statistical uncertainties from the near-site measurements.

\section{References}

[1] F. P. An et al. [Daya Bay Collaboration], Phys. Rev. Lett. 108 (2012) 171803.

[2] J. K. Ahn et al. [RENO Collaboration], Phys. Rev. Lett. 108 (2012) 191802.

[3] Y. Abe et al. [Double Chooz Collaboration], JHEP 1410 (2014) 086 [err: JHEP 1502 (2015) 074].

[4] K. Abe et al. [T2K Collaboration], Phys. Rev. Lett. 112 (2014) 061802

[5] P. Adamson et al. [MINOS Collaboration], Phys. Rev. Lett. 110 (2013) 17, 171801

[6] F. P. An et al. [Daya Bay Collaboration], Phys. Rev. Lett. 112 (2014) 061801.

[7] F. P. An et al. [Daya Bay Collaboration], Phys. Rev. Lett. 115 (2015), 111802.

[8] F. P. An et al. [Daya Bay Collaboration], arXiv:1508.04233.

[9] S. Seo (RENO Collaboration), in Neutrino2014: The XXVI International Conference on Neutrino Physics and Astrophysics, Boston, 2014 (unpublished).

[10] J. Beringer et al. (Particle Data Group), Phys. Rev. D 86, 010001 (2012), see Sec. 13.

[11] P. Adamson et al. (MINOS Collaboration), Phys. Rev. Lett. 112, 191801 (2014).

[12] K. Abe et al. (T2K Collaboration), Phys. Rev. Lett. 112, 181801 (2014). 